OPEN ACCESS

Edited by:

Fuchuan Li,

Shandong University, China

Reviewed by:

Inmaculada Navarro-Lérida, Autonomous University of Madrid,

Spain

Yong Fan,

Guangzhou Medical University, China

${ }^{*}$ Correspondence:

Shoko Nishihara

shoko@soka.ac.jp

Specialty section

This article was submitted to

Signaling,

a section of the journal

Frontiers in Cell and Developmental

Biology

Received: 30 June 2021 Accepted: 01 September 2021 Published: 23 September 2021

Citation:

Ogura C and Nishihara S (2021) Dermatan-4-O-Sulfotransferase-1 Contributes to the Undifferentiated

State of Mouse Embryonic Stem Cells. Front. Cell Dev. Biol. 9:733964.

doi: 10.3389/fcell.2021.733964

\section{Dermatan-4-0-Sulfotransferase-1 Contributes to the Undifferentiated State of Mouse Embryonic Stem Cells}

\author{
Chika Ogura ${ }^{1}$ and Shoko Nishihara ${ }^{1,2 *}$ \\ ' Department of Bioinformatics, Graduate School of Engineering, Soka University, Hachioji, Japan, ${ }^{2}$ Glycan and Life System \\ Integration Center (GaLSIC), Soka University, Hachioji, Japan
}

Mouse embryonic stem cells (mESCs) have the properties of self-renewal and pluripotency. Various signals and growth factors maintain their undifferentiated state and also regulate their differentiation. Glycosaminoglycans are present on the cell surface and in the cell matrix as proteoglycans. Previously, we and other groups reported that the glycosaminoglycan heparan sulfate contributes to both maintenance of undifferentiated state and regulation of mESC differentiation. It has been shown that chondroitin sulfate is needed for pluripotency and differentiation of mESCs, while keratan sulfate is a known marker of human ESCs or induced pluripotent stem cells. We also found that DS promotes neuronal differentiation from mESCs and human neural stem cells; however, the function of DS in the maintenance of mESCs has not yet been revealed. Here, we investigated the role of DS in mESCs by knockdown (KD) or overexpression (O/E) of the dermatan-4-O-sulfotransferase-1 (D4ST1) gene. We found that the activity of the ESC self-renewal marker alkaline phosphatase was reduced in D4ST1 KD mESCs, but, in contrast, increased in D4ST1 O/E mESCs. D4ST1 KD promoted endodermal differentiation, as indicated by an increase in $C d x 2$ expression. Conversely, Cdx2 expression was decreased by D4ST1 O/E. Wnt signaling, which is also involved in endodermal differentiation, was activated by D4ST1 KD and suppressed by D4ST1 O/E. Collectively, these results demonstrate that D4ST1 contributes to the undifferentiated state of mESCs. Our findings provide new insights into the function of DS in mESCs.

\section{Keywords: mouse embryonic stem cells, D4ST1, self-renewal, Cdx2, endodermal differentiation}

\section{INTRODUCTION}

Mouse embryonic stem cells (mESCs) are established from the inner cell mass at the blastocyst stage (Evans and Kaufman, 1981; Martin, 1981). They have the properties of self-renewal and pluripotency, which means that they are capable of differentiation into the three primary germ layers, endoderm, mesoderm, and ectoderm, via the epiblast and primitive endoderm. There are many studies showing that various signals and growth factors contribute to maintenance of undifferentiated state and regulation of differentiation in mESCs. Because the role of glycans in these processes has not been fully elucidated, we previously performed an RNA interference (RNAi) screen to identify glycosyltransferases essential for self-renewal and pluripotency in mESCs. 
To date, we have identified four glycan structures that are required to maintain the naïve pluripotent state: (1) LacdiNAc structure (GalNAc $\beta 1-4 G l c N A c)$ (Sasaki et al., 2011), (2) heparan sulfate (HS) (Sasaki et al., 2008, 2009; Hirano et al., 2012, 2013), (3) O-GlcNAc (Miura and Nishihara, 2016; Miura et al., 2018; Pecori et al., 2021), and (4) T antigen (Gal $\beta 1-3$ GalNAc) (Pecori et al., 2020).

Glycosaminoglycans (GAGs) such as HS are present on the cell surface and in the cell matrix as proteoglycans, consisting of GAG and a core protein. GAGs show diverse structures due to sulfation and have a characteristic disaccharide repeating structure. In addition to $\mathrm{HS}$, keratan sulfate (KS), and chondroitin sulfate (CS)/dermatan sulfate (DS) are wellknown GAGs. HS and CS/DS bind to the Ser residue of core proteins through a common linkage region, namely GlcA $\beta 1-3 \mathrm{Gal} \beta 1-3 \mathrm{Gal} \beta 1-4 \mathrm{Xyl} \beta-O-$-ser (Sugahara and Kitagawa, 2000), while KS binds to core proteins via an $N$-linked or O-linked oligosaccharide (Funderburgh, 2002). Previously, we and other groups showed that HS contributes to maintenance of undifferentiated state and regulation of differentiation in mESCs by promoting Wnt, BMP, FGF, and Fas signaling (Johnson et al., 2007; Sasaki et al., 2008, 2009; Kraushaar et al., 2010, 2012; Lanner et al., 2010; Fico et al., 2012; Hirano et al., 2012, 2013). In addition, Izumikawa et al. (2014) reported that CS is required for pluripotency and differentiation of mESCs, while KS is known as a marker of human ESCs or induced pluripotent stem cells (Andrews et al., 1984; Pera et al., 1988; Adewumi et al., 2007; Kawabe et al., 2013). To our knowledge, however, the function of DS in mESCs has not been revealed yet.

In the synthesis of DS, epimerization from glucuronic acid (GlcA) to iduronic acid (IdoA) is initially carried out by dermatan sulfate epimerase (Maccarana et al., 2006) or dermatan sulfate epimerase-like (Pacheco et al., 2009) after synthesis of the CS chain (i.e., GlcA-GalNAc repeating disaccharide structure). Subsequently, dermatan-4-O-sulfotransferase-1 (D4ST1) (Evers et al., 2001) transfers sulfate to the C-4 hydroxyl group of GalNAc. Lastly, sulfate is transferred to the C-6hydroxyl group of GalNAc and the C-2 hydroxyl group of IdoA by $\mathrm{N}$-acetylgalactosamine-4-sulfate 6-O-sulfotransferase (GalNAc4S-6ST) (Ito and Habuchi, 2000) and uronyl-2sulfotransferase (UST) (Kobayashi et al., 1999), respectively. While the GalNAc4S-6ST and UST sulfotransferases are common to both CS and DS, D4ST1 is specific to DS.

We previously reported that DS promotes neuronal differentiation from mESCs and human neural stem cells (Ogura et al., 2020). It is also known that D4ST1 is needed for neuronal differentiation from mouse neural stem cells (Bian et al., 2011). Moreover, D4ST1 deficiency is the cause of EhlersDanlos syndrome (EDS), a genetic connective tissue disorder with defects in skin, ligaments, articulation, internal organs, and blood vessels (Kosho, 2016; Malfait et al., 2017).

Here, therefore, we investigated the role of DS in the undifferentiated state of mESCs by knockdown or overexpression of D4ST1. We found that D4ST1 contributes to self-renewal of mESCs and D4ST1 knockdown induces endodermal differentiation by activating Wnt signaling. Our results provide new insights into function of DS in mESCs.

\section{MATERIALS AND METHODS}

\section{Cell Culture}

The R1 mESC line (Nagy et al., 1993) was cultured on mouse embryonic fibroblasts (MEFs) in mESC culture medium [DMEM (Gibco), 15\% FBS (Nichirei Bioscience, Inc.), 1\% penicillin/streptomycin (Gibco), $0.1 \mathrm{mM}$ 2-mercaptoethanol (Gibco), $1 \mathrm{mM}$ non-essential amino acids (Gibco), and 1,000 units/ml of LIF (Oriental Yeast)]. MEFs were isolated from embryos at E14.5 and inactivated by the addition of $10 \mu \mathrm{g} / \mathrm{ml}$ of mitomycin C (Sigma).

\section{Transfection}

For transient knockdown (KD) of D4ST1 in mESCs, we generated siRNA expression vectors using pSilencer 3.1-H1 (Ambion). The siRNA sequences used for RNAi were designed as described previously (Ui-Tei et al., 2004) by using siDirect ${ }^{1}$ :

E $g f p, 5^{\prime}$-GATCCCGCCACAACGTCTATATCATGGGGAAA ATCCATGATATAGACGTTGTGGCTTTTTTGGAAA-3'; D4S T1 KD1, 5'-GATCCCCAGCACTACTTCAAGTTCCTGTTTGG CTTCCTGTCACCAAACAGGAACTTGAAGTAGTGCTGTTT TTTA-3'; D4ST1 KD2, 5' -GATCCCTCCTCTTGCTAGGTCTGA ATCATTTGCTTCCTGTCACAAATGATTCAGACCTAGCAAG AGGATTTTTTA-3'; D4ST1 KD3， 5'-GATCCCCTTCAAGAT GTGCTACCTAAGGCTTCCTGTCACCTTAGGTAGCACATCT TGAAGTTTTTTA-3'. Egfp was used as a negative control.

We also generated a D4ST1 overexpression (O/E) vector using pCAGI-Puro (a kind gift of Professor Kumiko Ui-Tei). The vector was produced by using the $\mathrm{pGEM}^{\circledR}$-T Easy Vector Systems (Promega) as described previously (Kamiyama et al., 2006). We used an empty vector as a control for the O/E experiments.

Before transfection, we replated the mESCs at $1 \times 10^{6}$ cells on gelatin-coated $60-\mathrm{mm}$ culture dishes (NIPPON Genetics) containing LIF. After $16 \mathrm{~h}$, the cells were transfected with $4 \mu \mathrm{g}$ of siRNA expression vectors targeting D4ST1 (D4ST1 KD1 and D4ST1 KD2) or Egfp, or the D4ST1 O/E vector by using Lipofectamine 2000 (Invitrogen). At 1 day after transfection (TF day 1), transfected cells were selected by adding $2 \mu \mathrm{g} / \mathrm{ml}$ of puromycin (Sigma). We harvested the cells at TF day 2 for the D4ST1 O/E experiments or TF day 4 for the D4ST1 KD experiments.

\section{Cell Proliferation Assay}

D4ST1 KD mESCs at TF day 4 were replated at $8 \times 10^{3}$ cells per well on gelatin-coated 96-well plates (IWAKI) containing LIF. After $24 \mathrm{~h}$, we counted the number of viable cells by using microscopy.

\section{Alkaline Phosphatase Staining}

The transfected mESCs were replated at $1.25 \times 10^{5}$ cells per well on gelatin-coated 24-well plates (NIPPON Genetics) containing LIF. After 5 days, we carried out ALP staining with a StemTAG ${ }^{\text {TM }}$ Alkaline Phosphatase Staining Kit (Cell Biolabs, Inc.). ALPpositive colonies were counted by using microscopy.

\footnotetext{
${ }^{1}$ http://sidirect2.rnai.jp/
} 


\section{A}

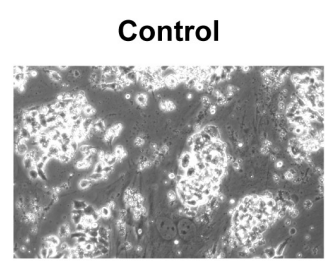

D4ST1 KD1

D4ST1 KD2
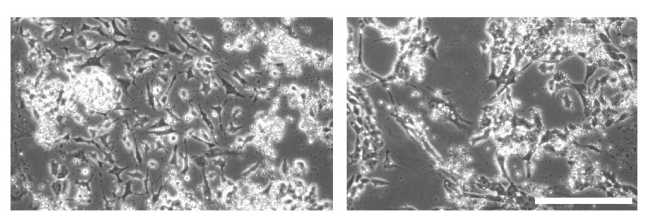

B

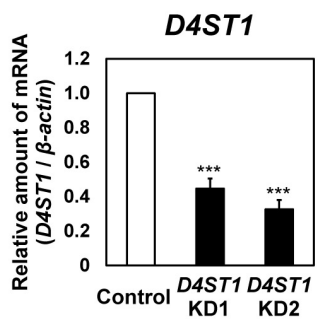

C

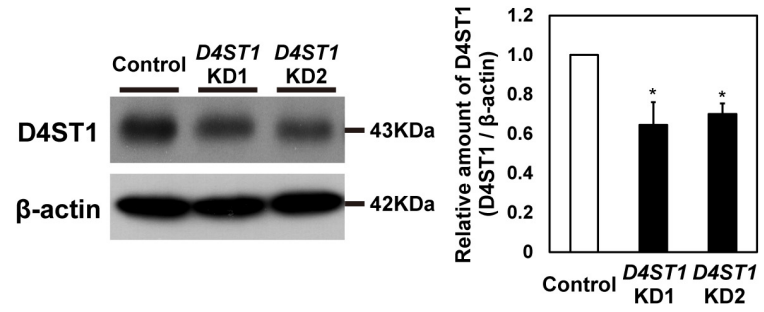

D
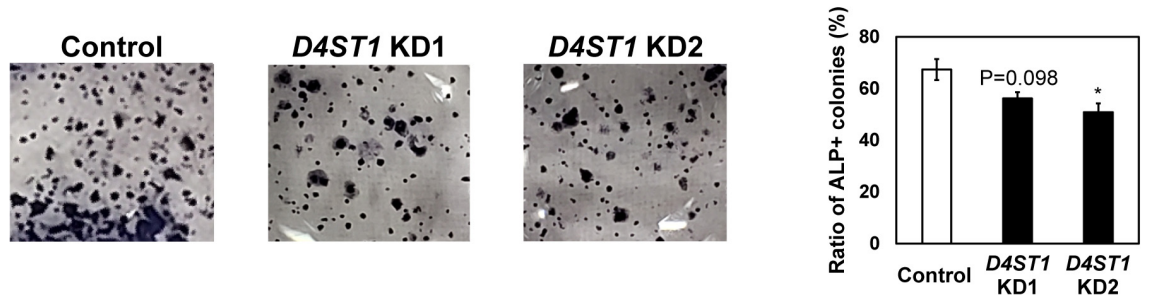

E
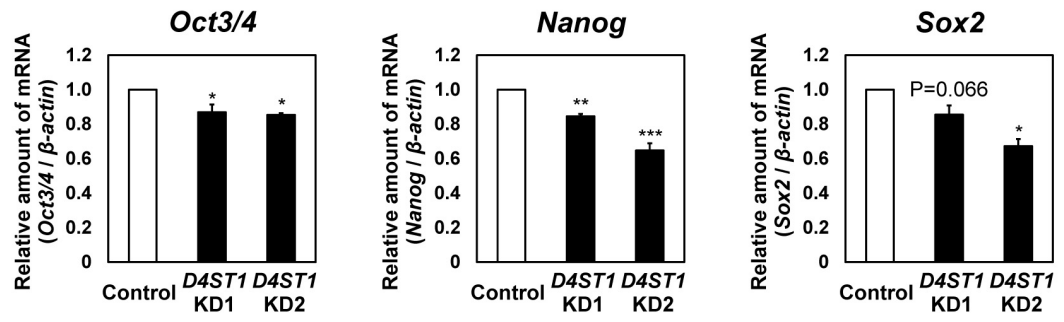

$\mathbf{F}$

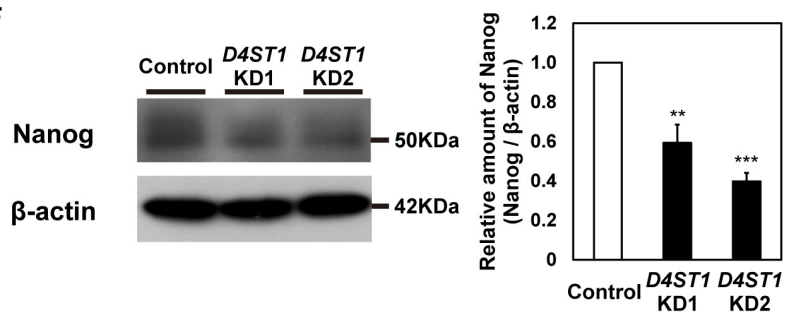

G
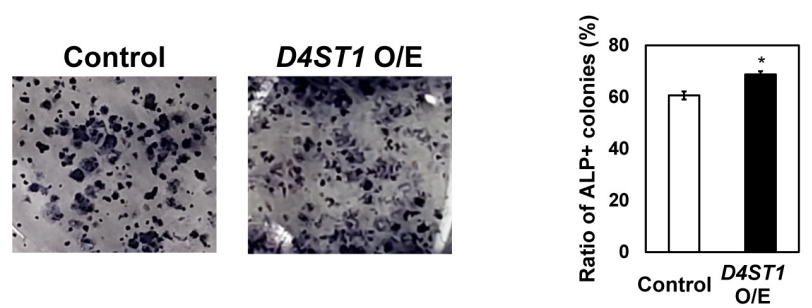

FIGURE 1 | (Continued) 
FIGURE 1 | D4ST1 contributes to self-renewal of mESCs. (A) Morphological observation of D4ST1 KD mESCs at day 4 after transfection (TF day 4). Scale bar: $200 \mu \mathrm{m}$. (B) Real-time PCR analysis of D4ST1 in D4ST1 KD mESCs at TF day 4. The amount of D4ST1 mRNA was normalized to that of $\beta$-actin mRNA and is shown relative to the control (set to 1). (C) Western blotting analysis of D4ST1 in D4ST1 KD mESCs at TF day 4. Histogram shows the mean densitometric readings of bands, which were normalized to $\beta$-actin and are shown relative to the control (set to 1). (D) Alkaline phosphatase (ALP) staining of D4ST1 KD mESCs at TF day 4. (Left) Representative images of ALP staining. (Right) Histogram showing the ratio of ALP-positive colonies. (E) Real-time PCR analysis of pluripotent markers in D4ST1 KD mESCs at TF day 4. The amounts of pluripotent marker mRNAs (Oct3/4, Nanog, and Sox2) were normalized to that of $\beta$-actin mRNA and are shown relative to the control (set to 1). (F) Western blotting analysis of Nanog in D4ST1 KD mESCs at TF day 4. Histogram shows the mean densitometric readings of bands, which were normalized to $\beta$-actin and are shown relative to the control (set to 1). The representative bands of the loading control ( $\beta$-actin) are the same as those in (C) because the same samples were used for these analyses. (G) ALP staining of D4ST1 O/E mESCs at TF day 2. (Left) Representative images of ALP staining. (Right) Histogram showing the ratio of ALP-positive colonies. The values shown are means $\pm S D(N=3)$. Those significantly different to the control by Dunnett test (B-F) or unpaired two-tailed Student's $t$-test (G) are indicated as follows: ${ }^{\star \star *} p<0.001 ;{ }^{\star \star} p<0.01 ;{ }^{*} p<0.05$.

\section{Real-Time PCR}

Total RNA was extracted from cells by using TRI Reagent ${ }^{\circledR}$ (Molecular Research Center, Inc.) and reverse-transcribed by using SuperScript ${ }^{\mathrm{TM}}$ VILO $^{\mathrm{TM}}$ Master Mix (Invitrogen). Realtime PCR was performed by using Quant Studio 12K Flex (Applied Biosystems). The relative amount of each mRNA was normalized against the amount of $\beta$-actin mRNA in the same sample. The primer sets for real-time PCR are listed in Supplementary Table 1.

\section{Western Blotting Analysis}

The transfected mESCs were lysed with lysis buffer $(50 \mathrm{mM}$ Tris-HCl pH 7.4, $150 \mathrm{mM} \mathrm{NaCl}, 1 \%$ Triton X-100, $5 \mathrm{mM}$ EDTA, $1 \mathrm{mM} \mathrm{Na} \mathrm{VO}_{4}, 10 \mathrm{mM} \mathrm{NaF}$, and protease inhibitors). The protein samples $(5-10 \mu \mathrm{g})$ were separated by $8 \%$ SDSPAGE and transferred to PVDF membranes (Millipore). After blocking with $1 \%$ BSA/TBST, the membranes were incubated with primary antibodies. The membranes were then incubated with secondary antibodies and Amersham ECL Prime Western Blotting Detection Reagent (GE Healthcare Life Science) was used for detection. The antibodies are listed in Supplementary Table 2.

\section{Statistical Analysis}

Data were compared with unpaired two-tailed Student's $t$-test or Dunnett test. Asterisks denote statistical significance (n.s., $p>0.05 ;{ }^{*} p<0.05$; $^{* *} p<0.01$; and $\left.{ }^{* * *} p<0.001\right)$.

\section{RESULTS}

\section{D4ST1 Contributes to Self-Renewal of mESCs}

To investigate function of DS in mESCs, we performed knockdown (KD) of D4ST1, which is the first sulfotransferase in the DS synthesis pathway (Figure 1A). We designed two constructs (D4ST1 KD1 and D4ST1 KD2), which expressed different siRNAs targeting D4ST1 mRNA, and one construct targeting Egfp as a negative control. After transfection of mESCs with these constructs, the decreased expression of D4ST1 mRNA and D4ST1 was confirmed by real-time PCR and western blotting, respectively (Figures 1B,C). Proliferation in D4ST1 KD mESCs was not changed as compared with control cells (Supplementary Figure 1). To determine selfrenewal potential, ALP staining was performed for the D4ST1
KD1 and KD2 transfected mESCs. The number of ALPpositive colonies was reduced by $D 4 S T 1 \mathrm{KD}$, indicating that D4ST1 contributes to self-renewal of mESCs (Figure 1D). Furthermore, the expression of three pluripotent markers, Oct3/4, Nanog and Sox2, were decreased in D4ST1 KD mESCs (Figure 1E and Supplementary Figure 2). The expression of Klf2 was decreased, while that of Klf4 and Rex1 did not change significantly (Supplementary Figure 3). The amount of Nanog was also significantly decreased in D4ST1 KD mESCs (Figure 1F).

We also examined the effect of overexpression $(\mathrm{O} / \mathrm{E})$ of D4ST1 in mESCs (Supplementary Figure 4A). The increased expression of D4ST1 mRNA and D4ST1 after transfection with the O/E vector was confirmed by real-time PCR and western blotting, respectively (Supplementary Figures 4B,C). In contrast to D4ST1 KD, D4ST1 O/E increased the number of ALP-positive colonies (Figure 1G), confirming that D4ST1 contributes to self-renewal of mESCs.

\section{Endodermal Differentiation of mESCs Is Induced by D4ST1 KD}

Next, we examined the expression of differentiation markers to determine which lineages are induced from mESCs by D4ST1 KD (Figure 2A and Supplementary Figure 2). In D4ST1 KD mESCs, the expression of two epiblast markers, Fgf5 and Otx2, was decreased. The expression of two mesoderm markers, $T$ and Mixl, was also significantly decreased. In contrast to mesodermal markers, the expression of two endoderm markers, Sox17 and Cdx2, was significantly increased in D4ST1 KD mESCs, indicating that the endodermal differentiation was induced in D4ST1 KD mESCs. Expression of the primitive endoderm marker Gata6 was significantly increased, indicating that the differentiation to primitive endoderm was also induced in D4ST1 KD mESCs. However, expression of the ectoderm marker Mash1 was not changed by D4ST1 KD. Collectively, these results suggest that D4ST1 contributes to the pluripotency of mESCs.

We also analyzed the expression of $C d x 2$, a marker of hindgut (Beck et al., 1995; Sherwood et al., 2007), in D4ST1 O/E mESCs. The expression of $C d x 2$ was significantly decreased in $D 4 S T 1 \mathrm{O} / \mathrm{E}$ mESCs (Figure 2B). It has been reported that $C d x 2$ expression is required for differentiation of hindgut (Stringer et al., 2008). Thus, the significantly increased or decreased expression of $C d x 2$ in the respective D4ST1 KD or D4ST1 O/E mESCs indicates 

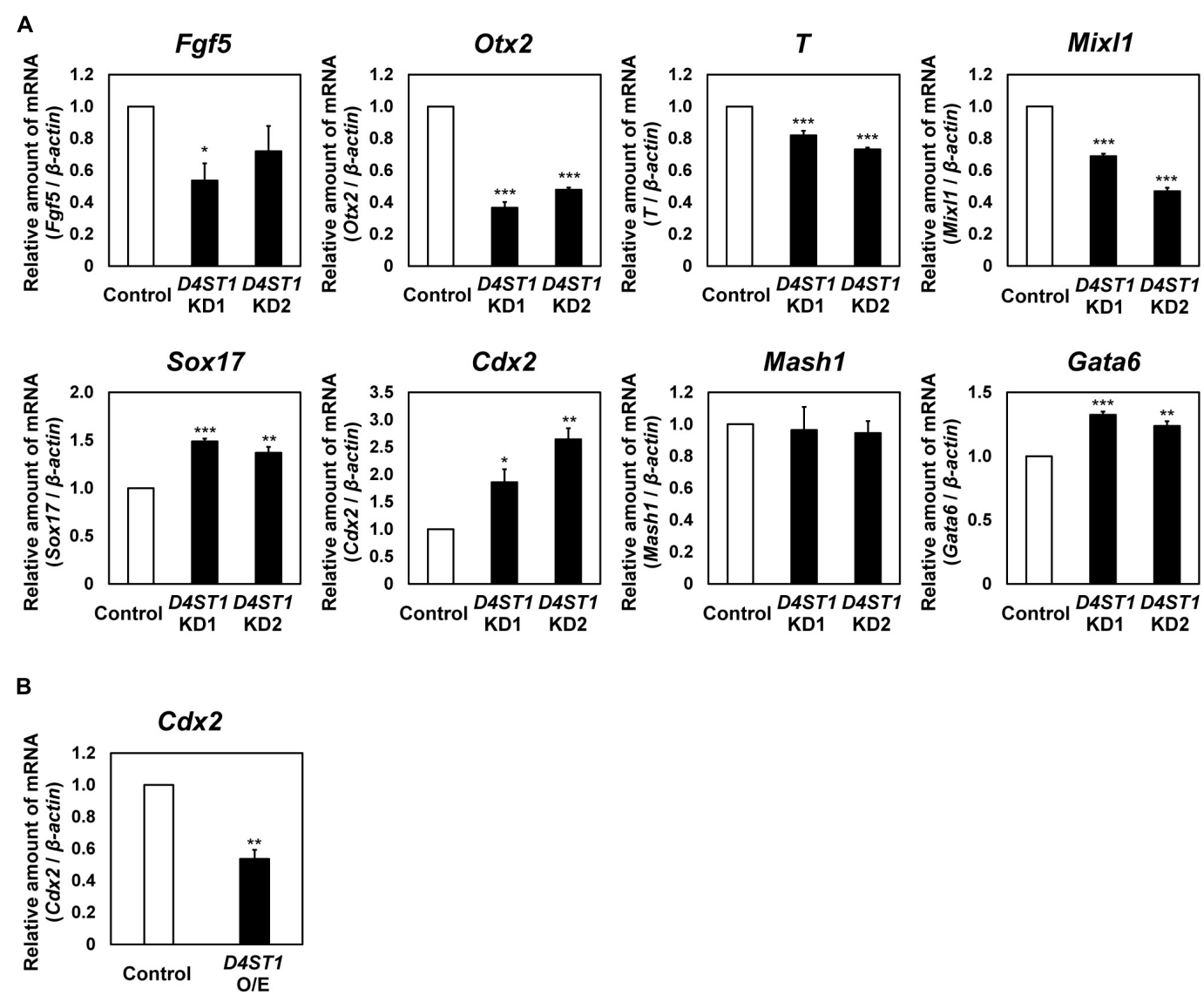

FIGURE 2 | Endodermal differentiation of mESCs is induced by D4ST1 KD. (A) Real-time PCR analysis of differentiation markers in D4ST1 KD at TF day 4. The amounts of differentiation marker mRNAs (Fgf5, Otx2, T, Mixl1, Sox17, Cdx2, Mash1, and Gata6) were normalized to that of $\beta$-actin mRNA and are shown relative to the control (set to 1). (B) Real-time PCR analysis of $C d x 2$ in D4ST1 O/E at TF day 2. The amount of Cdx2 mRNA was normalized to that of $\beta$-actin mRNA and is shown relative to the control (set to 1$)$. The values shown are means $\pm \mathrm{SD}(N=3)$. Those significantly different to the control by Dunnett test $(\mathbf{A})$ or unpaired two-tailed Student's $t$-test $(\mathbf{B})$ are indicated as follows: ${ }^{\star \star \star} p<0.001 ;{ }^{\star \star} p<0.01 ;{ }^{*} p<0.05$.

that D4ST1 might regulate endodermal differentiation, including differentiation to hindgut.

\section{BMP Signaling Is Suppressed and Wnt Signaling Is Activated by D4ST1 KD}

To analyze effect of D4ST1 KD on signaling pathways, we used western blotting to analyze several signaling components in D4ST1 KD mESCs. First, we examined the BMP/Smad1/5/8 signal, which contributes to self-renewal in mESCs by suppressing neural determination (Ying et al., 2003) and by up-regulating ERK-specific dual-specificity phosphatase 9 to reduce extracellular signal-regulated kinase activity, which is required for cell fate commitment (Li et al., 2012). Phosphorylated Smad1/5/8 was significantly decreased by D4ST1 KD (Figure 3A). Thus, the reduced activity of the ESC self-renewal marker ALP (Figure 1D) is caused by a decrease in BMP signal.

Second, we examined the $\mathrm{Wnt} / \beta$-catenin signal which induces endodermal differentiation (Zhong et al., 2017) and subsequently the hindgut domain during primitive gut tube formation in mouse (Engert et al., 2013). Whereas the relative amount of Active- $\beta$-catenin was significantly decreased by D4ST1 O/E (Figure 3C), it was significantly increased (Figure 3B) by D4ST1 KD. We also analyzed the expression of Wnt signaling target genes in D4ST1 O/E mESCs; Lef1 was significantly decreased, while that of Axin2 and $C d x 1$ tended to be decreased (Supplementary Figure 5). These results demonstrate that D4ST1 KD induces endodermal differentiation and subsequent regionalization of the hindgut domain by activating Wnt signaling.

\section{DISCUSSION}

In this study, we found that self-renewal and the undifferentiated state of mESCs were compromised by D4ST1 KD. In D4ST1 KD mESCs, self-renewal of mESCs was reduced and endodermal differentiation was induced. In particular, the expression of $C d x 2$, which is a hindgut marker, was significantly increased by D4ST1 KD and significantly decreased by D4ST1 O/E. Similarly, Wnt signal was activated by D4ST1 KD and suppressed by D4ST1 O/E. 
A
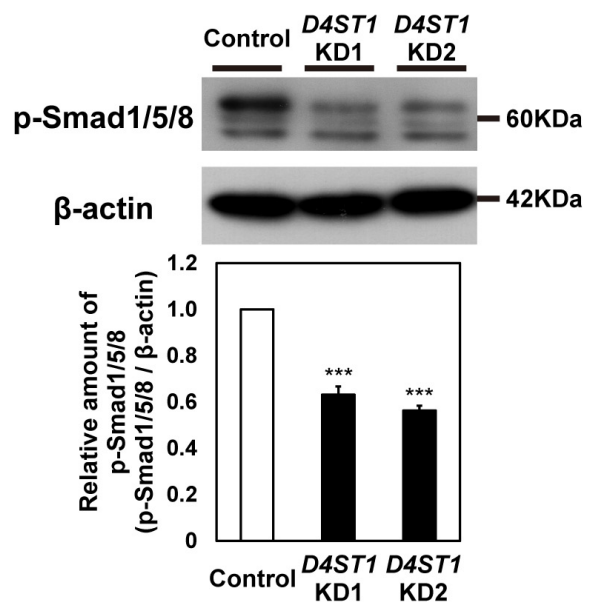

B

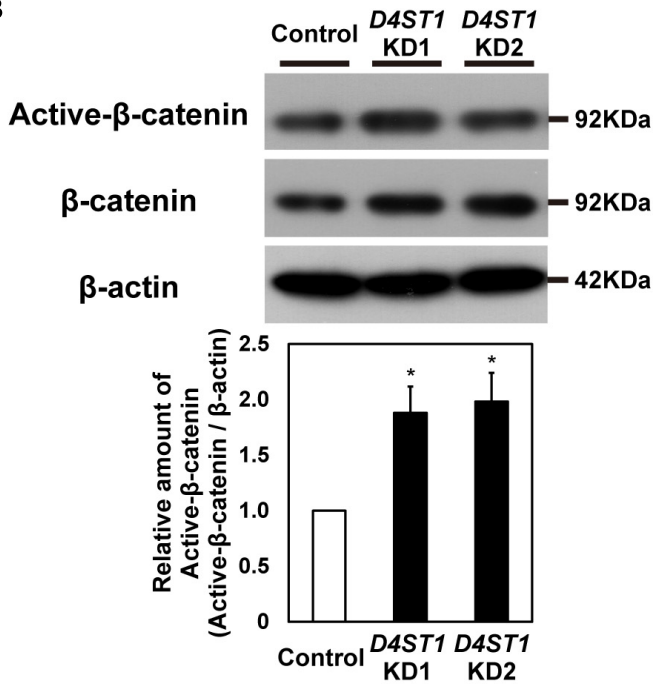

C
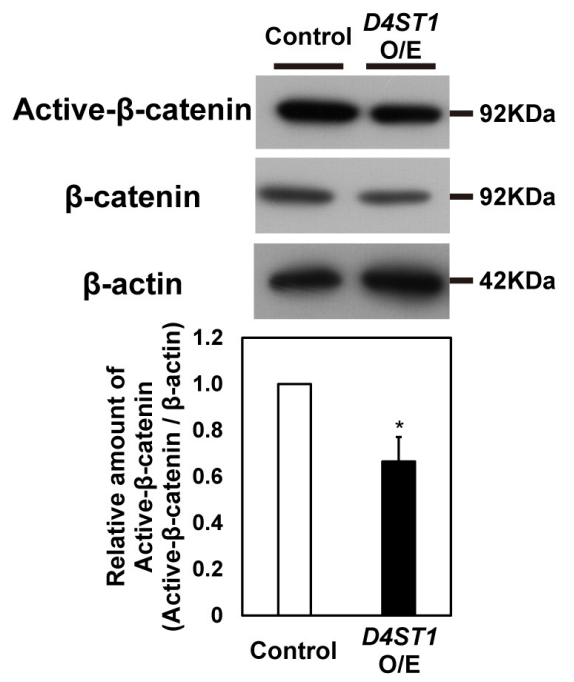

FIGURE 3 | (Continued)
FIGURE 3 | Wht signaling is activated by D4ST1 KD. (A,B) Western blotting analysis of $p$-Smad $1 / 5 / 8$ and Active- $\beta$-catenin in D4ST1 KD mESCs at TF day 4. Histogram shows the mean densitometric readings of bands normalized to $\beta$-actin, and was shown relative to the control (set to 1). The representative bands of the loading control ( $\beta$-actin) are the same as those in Figure 1C because the same samples were used for these analyses. (C) Western blotting analysis of Active- $\beta$-catenin in D4ST1 O/E mESCs at TF day 2. Histogram shows the mean densitometric readings of bands, which were normalized to $\beta$-catenin and are shown relative to the control (set to 1). The values shown are means $\pm S D(N=3)$. Those significantly different to the control by Dunnett test $(\mathbf{A}, \mathbf{B})$ or unpaired two-tailed Student's $t$-test $\mathbf{( C )}$ are indicated as follows: ${ }^{* \star *} p<0.001 ;{ }^{*} p<0.05$.

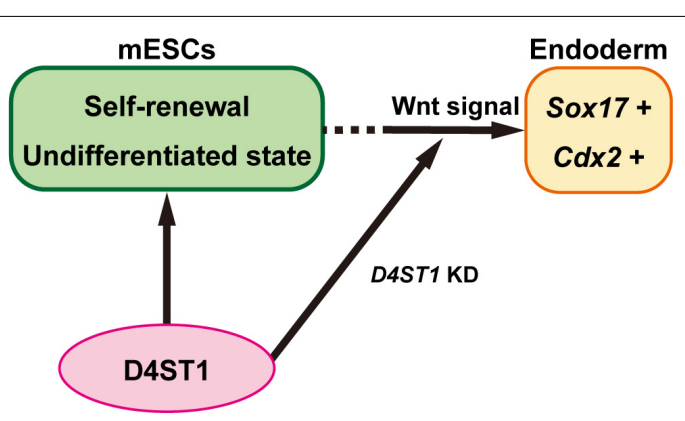

FIGURE 4 | D4ST1 contributes to the undifferentiated state of mESCs. D4ST1 contributes to self-renewal and the undifferentiated state of mESCs. Knockdown of D4ST1 causes promotion of endodermal differentiation by activation of Wnt signaling.

It has been reported that $C d x 2$ is essential for determination of intestinal mesoderm or endoderm differentiation (Stringer et al., 2008). The endoderm and mesoderm arise from a transient common precursor cell population referred to as "mesendoderm." The specification of endoderm requires $W n t / \beta$-catenin signaling, which maintains the expression of Nodal, which in turn promotes the expression of a network of transcription factors within the endodermal lineage including Sox17 (Zorn and Wells, 2009). After endodermal linage determination, the gut tube is formed. The gut tube then becomes regionalized along the dorsal-ventral and anteriorposterior axes into broad foregut, midgut, and hindgut domains (Zorn and Wells, 2009). The Wnt/ $\beta$-catenin signal also specifies the hindgut domain by inducing $C d x 2$ expression, which is required for both the hindgut and positioning of the foreguthindgut boundary in mouse development (Sherwood et al., 2011). Therefore, our results demonstrate that D4ST1 contributes to the undifferentiated state of mESCs, and D4ST1 KD induces endodermal and subsequent hindgut differentiation by activating Wnt signaling (Figure 4).

CDX2 is also required for intestinal development in human pluripotent stem cells, and WNT signaling similarly promotes endoderm-hindgut differentiation (Kumar et al., 2019). Although further investigation is needed to elucidate the function of D4ST1 in human development, D4ST1 deficiency is known to be one of the causes of EDS (Kosho, 2016). D4ST1-deficient EDS presents characteristic craniofacial features, multiple congenital contractures, and progressive joint and skin laxity. Of note, 
joints and dermis are tissues derived from mesoderm. In the present study, in contrast to endodermal markers, mesodermal markers were suppressed by D4ST1 KD. Thus, D4ST1 may contribute to mesodermal differentiation in humans. In addition, it has been reported that skin complaints are caused by disorganization of collagen networks due to decorin, a dermatan sulfate proteoglycan (DSPG) (Hirose et al., 2018). There are several DSPGs, including decorin, biglycan, and fibromodulin. Determination of the DSPG that contributes to differentiation of mESCs will be an interesting issue for future study.

Because D4ST1 is a sulfotransferase involved in DS synthesis, it is possible that DS regulates Wnt signaling. GAGs such as HS and CS play a key role in signal transduction as co-receptors or as trappers by binding signal ligands (Wang et al., 2017). For example, DS has been reported to interact with bFGF, FGF7, and EGF (Taylor et al., 2005; Bian et al., 2011). To our knowledge, however, binding of DS to Wnt has not been demonstrated. This is also an interesting issue for future analysis.

In conclusion, we have shown that D4ST1 is required for self-renewal and the undifferentiated state in mESCs and D4ST $\mathrm{KD}$ induces endoderm differentiation and subsequent hindgut differentiation by activating Wnt signals. This study provides new insights into function of DS in mESCs.

\section{DATA AVAILABILITY STATEMENT}

The original contributions presented in the study are included in the article/Supplementary Material, further inquiries can be directed to the corresponding author.

\section{REFERENCES}

Adewumi, O., Aflatoonian, B., Ahrlund-Richter, L., Amit, M., Andrews, P. W., Beighton, G., et al. (2007). Characterization of human embryonic stem cell lines by the International Stem Cell Initiative. Nat. Biotechnol. 25, 803-816. doi: $10.1038 / n b t 1318$

Andrews, P. W., Banting, G., Damjanov, I., Arnaud, D., and Avner, P. (1984). Three monoclonal antibodies defining distinct differentiation antigens associated with different high molecular weight polypeptides on the surface of human embryonal carcinoma cells. Hybridoma 3, 347-361.

Beck, F., Erler, T., Russell, A., and James, R. (1995). Expression of Cdx-2 in the mouse embryo and placenta: possible role in patterning of the extra-embryonic membranes. Dev. Dynam. 204, 219-227. doi: 10.1002/aja.1002040302

Bian, S., Akyüz, N., Bernreuther, C., Loers, G., Laczynska, E., Jakovcevski, I., et al. (2011). Dermatan sulfotransferase Chst14/D4st1, but not chondroitin sulfotransferase Chst11/C4st1, regulates proliferation and neurogenesis of neural progenitor cells. J. Cell Sci. 124, 4051-4063. doi: 10.1242/jcs.088120

Engert, S., Burtscher, I., Liao, W. P., Dulev, S., Schotta, G., and Lickert, H. (2013). Wnt/ $\beta$-catenin signalling regulates Sox 17 expression and is essential for organizer and endoderm formation in the mouse. Development 140, 3128-3138. doi: 10.1242/dev.088765

Evans, M. J., and Kaufman, M. H. (1981). Establishment in culture of pluripotential cells from mouse embryos. Nature 292, 154-156. doi: 10.1038/292154a0

Evers, M. R., Xia, G., Kang, H.-G., Schachner, M., and Baenziger, J. U. (2001). Molecular cloning and characterization of a dermatan-specific $\mathrm{N}$-acetylgalactosamine 4-O-sulfotransferase. J. Biol. Chem. 276, 36344-36353. doi: 10.1074/jbc.m105848200

Fico, A., Chevigny, A. D., Egea, J., Bösl, M. R., Cremer, H., Maina, F., et al. (2012). Modulating glypican4 suppresses tumorigenicity of embryonic stem

\section{AUTHOR CONTRIBUTIONS}

$\mathrm{CO}$ and $\mathrm{SN}$ : conceptualization. CO: methodology, validation, formal analysis, investigation, data curation, writing - original draft, and visualization. SN: resources, writing - review and editing, supervision, project administration, and funding acquisition. Both authors contributed to the article and approved the submitted version.

\section{FUNDING}

This work was supported by Japan Society for the Promotion of Science (JSPS) KAKENHI Grant Number JP18K06139 (to SN) and Japan Science and Technology Agency (JST)-Mirai Program Grant Number JPMJMI18GB (to SN).

\section{ACKNOWLEDGMENTS}

We thank Kumiko Ui-Tei for providing pCAGI-Puro. We also thank Taichi Miura for the constructive discussion and advice.

\section{SUPPLEMENTARY MATERIAL}

The Supplementary Material for this article can be found online at: https://www.frontiersin.org/articles/10.3389/fcell.2021. 733964/full\#supplementary-material

cells while preserving self-renewal and pluripotency. Stem Cells 30, 1863-1874. doi: 10.1002/stem.1165

Funderburgh, J. L. (2002). Keratan sulfate biosynthesis. Iubmb Life 54, 187-194. doi: $10.1080 / 15216540214932$

Hirano, K., Kuppevelt, T. H. V., and Nishihara, S. (2013). The transition of mouse pluripotent stem cells from the naïve to the primed state requires Fas signaling through 3-O sulfated heparan sulfate structures recognized by the HS4C3 antibody. Biochem. Bioph. Res. Co. 430, 1175-1181. doi: 10.1016/j.bbrc.2012.12. 005

Hirano, K., Sasaki, N., Ichimiya, T., Miura, T., Kuppevelt, T. H. V., and Nishihara, S. (2012). 3-O-sulfated heparan sulfate recognized by the antibody HS4C3 contribute to the differentiation of mouse embryonic stem cells via Fas signaling. PLoS One 7:e43440. doi: 10.1371/journal.pone.0043440

Hirose, T., Takahashi, N., Tangkawattana, P., Minaguchi, J., Mizumoto, S., Yamada, S., et al. (2018). Structural alteration of glycosaminoglycan side chains and spatial disorganization of collagen networks in the skin of patients with mcEDSCHST14. Biochimica Et Biophysica Acta Bba - Gen Subj 1863, 623-631. doi: 10.1016/j.bbagen.2018.12.006

Ito, Y., and Habuchi, O. (2000). Purification and characterization of $\mathrm{N}$-acetylgalactosamine 4-sulfate 6-O-sulfotransferase from the squid cartilage. J. Biol. Chem. 275, 34728-34736. doi: 10.1074/jbc.m909633199

Izumikawa, T., Sato, B., and Kitagawa, H. (2014). Chondroitin sulfate is indispensable for pluripotency and differentiation of mouse embryonic stem cells. Sci. Rep. 4:3701. doi: 10.1038/srep03701

Johnson, C. E., Crawford, B. E., Stavridis, M., Dam, G., Wat, A. L., Rushton, G., et al. (2007). Essential alterations of heparan sulfate during the differentiation of embryonic stem cells to Sox1-enhanced green fluorescent protein-expressing neural progenitor cells. Stem Cells 25, 2389-2389. doi: 10.1634/stemcells.20060445erratum 
Kamiyama, S., Sasaki, N., Goda, E., Ui-Tei, K., Saigo, K., Narimatsu, H., et al. (2006). Molecular cloning and characterization of a novel $3^{\prime}$-phosphoadenosine 5'-phosphosulfate transporter, PAPST2. J. Biol. Chem. 281, 10945-10953. doi: 10.1074/jbc.m508991200

Kawabe, K., Tateyama, D., Toyoda, H., Kawasaki, N., Hashii, N., Nakao, H., et al. (2013). A novel antibody for human induced pluripotent stem cells and embryonic stem cells recognizes a type of keratan sulfate lacking oversulfated structures. Glycobiology 23, 322-336. doi: 10.1093/glycob/cws159

Kobayashi, M., Sugumaran, G., Liu, J., Shworak, N. W., Silbert, J. E., and Rosenberg, R. D. (1999). Molecular cloning and characterization of a human uronyl 2-sulfotransferase that sulfates iduronyl and glucuronyl residues in dermatan/chondroitin sulfate. J. Biol. Chem. 274, 10474-10480. doi: 10.1074/ jbc.274.15.10474

Kosho, T. (2016). CHST14/D4ST1 deficiency: new form of Ehlers-Danlos syndrome. Pediatr. Int. 58, 88-99. doi: 10.1111/ped.12878

Kraushaar, D. C., Rai, S., Condac, E., Nairn, A., Zhang, S., Yamaguchi, Y., et al. (2012). Heparan sulfate facilitates FGF and BMP signaling to drive mesoderm differentiation of mouse embryonic stem cells. J. Biol. Chem. 287, 22691-22700. doi: 10.1074/jbc.m112.368241

Kraushaar, D. C., Yamaguchi, Y., and Wang, L. (2010). Heparan sulfate is required for embryonic stem cells to exit from self-renewal. J. Biol. Chem. 285, 59075916. doi: 10.1074/jbc.m109.066837

Kumar, N., Tsai, Y.-H., Chen, L., Zhou, A., Banerjee, K. K., Saxena, M., et al. (2019). The lineage-specific transcription factor CDX2 navigates dynamic chromatin to control distinct stages of intestine development. Development 146:dev172189. doi: 10.1242/dev.172189

Lanner, F., Lee, K. L., Sohl, M., Holmborn, K., Yang, H., Wilbertz, J., et al. (2010). Heparan sulfation-dependent fibroblast growth factor signaling maintains embryonic stem cells primed for differentiation in a heterogeneous state. Stem Cells 28, 191-200. doi: 10.1002/stem.265

Li, Z., Fei, T., Zhang, J., Zhu, G., Wang, L., Lu, D., et al. (2012). BMP4 signaling acts via dual-specificity phosphatase 9 to control ERK activity in mouse embryonic stem cells. Cell Stem Cell 10, 171-182. doi: 10.1016/j.stem.2011.12.016

Maccarana, M., Olander, B., Malmström, J., Tiedemann, K., Aebersold, R., Lindahl, U., et al. (2006). Biosynthesis of dermatan sulfate chondroitin-glucuronate C5epimerase is identical to SART2. J. Biol. Chem. 281, 11560-11568. doi: 10.1074/ jbc.m513373200

Malfait, F., Francomano, C., Byers, P., Belmont, J., Berglund, B., Black, J., et al. (2017). The 2017 international classification of the Ehlers-Danlos syndromes. Am. J. Med. Genet. Part C Semin. Med. Genet. 175, 8-26. doi: 10.1002/ajmg.c. 31552

Martin, G. R. (1981). Isolation of a pluripotent cell line from early mouse embryos cultured in medium conditioned by teratocarcinoma stem cells. Proc. Natl. Acad. Sci. 78, 7634-7638. doi: 10.1073/pnas.78.12.7634

Miura, T., Kume, M., Kawamura, T., Yamamoto, K., Hamakubo, T., and Nishihara, S. (2018). O-GlcNAc on PKC $\zeta$ inhibits the FGF4-PKC $\zeta-M E K-E R K 1 / 2$ pathway via inhibition of PKCऽ phosphorylation in mouse embryonic stem cells. Stem Cell Rep. 10, 272-286. doi: 10.1016/j.stemcr.2017.11.007

Miura, T., and Nishihara, S. (2016). O-GlcNAc is required for the survival of primed pluripotent stem cells and their reversion to the naïve state. Biochem. Bioph. Res. Co. 480, 655-661. doi: 10.1016/j.bbrc.2016.10.111

Nagy, A., Rossant, J., Nagy, R., Abramow-Newerly, W., and Roder, J. C. (1993). Derivation of completely cell culture-derived mice from early-passage embryonic stem cells. Proc. Natl. Acad. Sci. 90, 8424-8428. doi: 10.1073/pnas. 90.18 .8424

Ogura, C., Hirano, K., Mizumoto, S., Yamada, S., and Nishihara, S. (2020). Dermatan sulphate promotes neuronal differentiation in mouse and human stem cells. J. Biochem. 169, 55-64. doi: 10.1093/jb/mvaa087

Pacheco, B., Malmström, A., and Maccarana, M. (2009). Two dermatan sulfate epimerases form iduronic acid domains in dermatan sulfate. J. Biol. Chem. 284, 9788-9795. doi: 10.1074/jbc.m809339200

Pecori, F., Akimoto, Y., Hanamatsu, H., Furukawa, J., Shinohara, Y., Ikehara, Y., et al. (2020). Mucin-type O-glycosylation controls pluripotency in mouse embryonic stem cells via Wnt receptor endocytosis. J. Cell Sci. 133:jcs245845. doi: $10.1242 /$ jcs. 245845

Pecori, F., Kondo, N., Ogura, C., Miura, T., Kume, M., Minamijima, Y., et al. (2021). Site-specific O-GlcNAcylation of Psme3 maintains mouse stem cell pluripotency by impairing P-body homeostasis. Cell Rep. 36, 109361. doi: 10. 1016/j.celrep.2021.109361
Pera, M. F., Blasco-Lafita, M. J., Cooper, S., Mason, M., Mills, J., and Monaghan, P. (1988). Analysis of cell-differentiation lineage in human teratomas using new monoclonal antibodies to cytostructural antigens of embryonal carcinoma cells. Differentiation 39, 139-149. doi: 10.1111/j.1432-0436.1988.tb 00089.x

Sasaki, N., Hirano, T., Ichimiya, T., Wakao, M., Hirano, K., Kinoshita-Toyoda, A., et al. (2009). The $3^{\prime}$-phosphoadenosine $5^{\prime}$-phosphosulfate transporters, PAPST1 and 2, contribute to the maintenance and differentiation of mouse embryonic stem cells. PLoS One 4:e8262. doi: 10.1371/journal.pone.0008262

Sasaki, N., Okishio, K., Ui-Tei, K., Saigo, K., Kinoshita-Toyoda, A., Toyoda, H., et al. (2008). Heparan sulfate regulates self-renewal and pluripotency of embryonic stem cells. J. Biol. Chem. 283, 3594-3606. doi: 10.1074/jbc. m705621200

Sasaki, N., Shinomi, M., Hirano, K., Ui-Tei, K., and Nishihara, S. (2011). LacdiNAc (GalNAc $\beta 1-4 \mathrm{GlcNAc}$ ) contributes to self-renewal of mouse embryonic stem cells by regulating leukemia inhibitory factor/STAT3 signaling. Stem Cells 29, 641-650. doi: 10.1002/stem.615

Sherwood, R. I., Jitianu, C., Cleaver, O., Shaywitz, D. A., Lamenzo, J. O., Chen, A. E., et al. (2007). Prospective isolation and global gene expression analysis of definitive and visceral endoderm. Dev. Biol. 304, 541-555. doi: 10.1016/j.ydbio. 2007.01.011

Sherwood, R. I., Maehr, R., Mazzoni, E. O., and Melton, D. A. (2011). Wnt signaling specifies and patterns intestinal endoderm. Mech. Dev. 128, 387-400. doi: 10.1016/j.mod.2011.07.005

Stringer, E. J., Pritchard, C. A., and Beck, F. (2008). Cdx2 initiates histodifferentiation of the midgut endoderm. Febs. Lett. 582, 2555-2560. doi: 10.1016/j.febslet.2008.06.024

Sugahara, K., and Kitagawa, H. (2000). Recent advances in the study of the biosynthesis and functions of sulfated glycosaminoglycans. Curr. Opin. Struc. Biol. 10, 518-527. doi: 10.1016/s0959-440x(00)00125-1

Taylor, K. R., Rudisill, J. A., and Gallo, R. L. (2005). Structural and sequence motifs in dermatan sulfate for promoting fibroblast growth factor-2 (FGF2) and FGF-7 activity. J. Biol. Chem. 280, 5300-5306. doi: 10.1074/jbc.m410 412200

Ui-Tei, K., Naito, Y., Takahashi, F., Haraguchi, T., Ohki-Hamazaki, H., Juni, A., et al. (2004). Guidelines for the selection of highly effective siRNA sequences for mammalian and chick RNA interference. Nucleic Acids Res. 32, 936-948. doi: 10.1093/nar/gkh247

Wang, M., Liu, X., Lyu, Z., Gu, H., Li, D., and Chen, H. (2017). Glycosaminoglycans (GAGs) and GAG mimetics regulate the behavior of stem cell differentiation. Coll. Surf. B Biointerf. 150, 175-182. doi: 10.1016/j.colsurfb.2016.11.022

Ying, Q. L., Nichols, J., Chambers, I., and Smith, A. (2003). BMP induction of Id proteins suppresses differentiation and sustains embryonic stem cell selfrenewal in collaboration with STAT3. Cell 115, 281-292. doi: 10.1016/s00928674(03)00847-x

Zhong, W., Lai, Y., Yu, T., Xia, Z. S., Yuan, Y. H., Ouyang, H., et al. (2017). Wnt and Nodal signaling simultaneously induces definitive endoderm differentiation of mouse embryonic stem cells. Romanian J. Morphol. Embryology Revue Roumaine De Morphol Et Embryologie 58, 527-535.

Zorn, A. M., and Wells, J. M. (2009). Vertebrate endoderm development and organ formation. Cell Dev. Biol. 25, 221-251. doi: 10.1146/annurev.cellbio.042308. 113344

Conflict of Interest: The authors declare that the research was conducted in the absence of any commercial or financial relationships that could be construed as a potential conflict of interest.

Publisher's Note: All claims expressed in this article are solely those of the authors and do not necessarily represent those of their affiliated organizations, or those of the publisher, the editors and the reviewers. Any product that may be evaluated in this article, or claim that may be made by its manufacturer, is not guaranteed or endorsed by the publisher.

Copyright (C) 2021 Ogura and Nishihara. This is an open-access article distributed under the terms of the Creative Commons Attribution License (CC BY). The use, distribution or reproduction in other forums is permitted, provided the original author(s) and the copyright owner(s) are credited and that the original publication in this journal is cited, in accordance with accepted academic practice. No use, distribution or reproduction is permitted which does not comply with these terms. 